\title{
Multi-Objective Optimization of Motor Vessel Route
}

\author{
Stéphane Marie and Eric Courteille \\ Université Européenne de Bretagne, France, \\ INSA-LGCGM - EA 3913, 20, avenue des Buttes de Coësmes 35043 RENNES Cédex \\ stephane.marie@insa-rennes.fr \\ eric.courteille@insa-rennes.fr
}

\begin{abstract}
This paper presents an original method that allows computation of the optimal route of a motor vessel by minimizing its fuel consumption. The proposed method is based on a new and efficient meshing procedure that is used to define a set of possible routes. A consumption prediction tool has been developed in order to estimate the fuel consumption along a given trajectory. The consumption model involves the effects of the meteorological conditions, the shape of the hull and the power train characteristics. Pareto-optimization with a Multi-Objective Genetic Algorithm (MOGA) is taken as a framework for the definition and the solution of the multi-objective optimization problem addressed. The final goal of this study is to provide a decision helping tool giving the route that minimizes the fuel consumption in a limited or optimum time.
\end{abstract}

\section{INTRODUCTION}

Ship weather routing develops an optimum track for ocean voyages based on forecasts of weather, sea conditions, and ship's characteristics. Within specified limits of weather and sea conditions, the term optimum is used to mean maximum safety and crew confort, minimum fuel and oil consumption, minimum time or distance underway, or any desired combination of these factors.

There exists many works dealing with the problem of optimal ship weather routing, in which motor or sailing vessels are considered. The approaches in the literature may be divided into four categories : isochrone construction, application of the calculus of variation, dynamic programming and evolutionary algorithms.

James (1957) proposed a scheme for non-variable weather conditions using lines of equal-time, or isochrones to achieve minimal-time objective. This method is very efficient for solving the problem of determinist minimal-time weather routing but is not adapted for minimization of the vessel's fuel consumption. Numerous variation of this type of method have been presented. For exemple Hagiwara \& Spaans (1987) presented an improvement of isochrone definition for sail-assisted motor vessel routing. In addition to time objective, the author tried to minimize fuel consumption but the method is not very efficient because the propeller speed is kept constant on the track.

Haltiner, Hamilton and Árnason (1962) solved the same problem by using the calculus of variations. This method is based on parametric curves obtained by solving the associated Euler differential equation by relaxation methods. Bleick $\&$ Faulkner (1965) extended this approach to the case of deterministic variations of sea state. This definition is mathematicaly elegant but impractical for ship weather routing because of convergence problems.

The third approach was proposed by Zappoli (1972), who treated the minimal time problem as a decision process solvable using dynamic programming. The sailing domain is discretized using grid refinement techniques. Allsopp \& al. (2000), modified this method integrating branching scenario structure to model the manner the weather will evolve in time. The main advantage of that kind of method is that the problem is divided into a set of linked stages and the optimal decision depends on decisions made in the previous stages. But, for fine grid, the calculation time may be very high and the amount of data very large.

The most recent approaches use B-spline technics and evolutionary algorithms in order to minimize fuel consumption and maximize safety. Harries, Heimann and Hinnenthal (2003) proposed such a 
method for large motor vessels using MultiObjective Genetic Algorithms. Hinnethal and Saertra (2005) improved this method considering the stochastic nature of weather along the route. Böttner (2007) recently used this work combined with dynamic programming for costal approach in order to propose the best possible track from harbor to harbor. These methods are characterized by a low number of free variables to describe both the course and the speed of the boat. Moreover, a high number of route variants can be considered from which Pareto optimal solutions may be identified.

In the four different approaches, the scheme for optimisation is almost the same :

- Mathematical modeling of the ship to compute the objectives,

- 3-D interpolation (time and space) of weather and sea state data,

- Parametric route definition,

- Optimization of the route using an algorithm.

Our work is based on a new discretization of the research space based on few physical parameters. This parametric definition of the griding makes it understandable and easy to tune. Moreover this kind of meshing may be applicable for all type of vessels, journeys and all weather conditions are easily taken into account. The gridding of the sail area is systematic and uniform since it is based on spherical geometry and accepts constrains like bathymetric data. Our work is related to the method proposed by Harries \& al. because we kept their definition of both the geography and speed since it allows the complete location of the boat in space and time. The modeling of motor vessels is not the purpose of this work and will just be briefly presented. Future works concern the identification of the vessel model using fuzzy logic technique in order to obtain an accurate consumption model. Since this identification is not achieved yet, a model from the literature will be used to present this new gridding method.

The meshing of the explored area is presented first. Then, the way of constructing routes is shown and a sensitivity of the meshing is presented. Next, the way of modeling a motor vessel is introduced. Finally, results of numerical optimizations are presented in order to evaluate the limitations and benefits of the proposed meshing method.

\section{MESHING OF THE EXPLORED AREA}

The new automatic meshing method that we propose is based on spherical rhombus where two of the opposite vertexes are the departure and the arrival points. The main advantages of this discretization of the sailing area are :

- The genericity of its construction taking into account the sea-beds geography, the time dependant meteorological data and the characteristics of the vessel.

- The systematic gridding of the explored area with few physical parameters.

The automation of its calculation leading to optimizable routes.

- The possible reactualization of the rhombus to change the routing policy during the sailing.

\subsection{Rhombus definition}

In this part $M$ denotes the departure point, $M^{\prime}$ the arrival one and $O$ is the center of Earth considered spherical. $\left(P_{1}\right)$ is the plane containing the lines $(O M)$ and $\left(O M^{\prime}\right)$ carrying respectively vectors $\mathbf{i}$ and j. $\gamma$ is the angle between these two vectors and $\mathbf{k}$ is their bisectrix. $\mathbf{w}$ is one vector normal both to $\mathbf{i}$ and j. We also define the two related unit vectors $\mathbf{k}_{u}$ and $\mathbf{w}_{u}$. Let be $\left(P_{2}\right)$ the plane containing $\mathbf{k}, \mathbf{w}$, the two remain vertexes of the rhombus $A$ and $B$ and point $O$. The lines $(O A)$ and $(O B)$ are directed by vectors $\mathbf{a}$ and $\mathbf{b}$. We defined $(\mathbf{a}, \mathbf{b})=2 \delta$. This notation is recalled in the figure 1 .

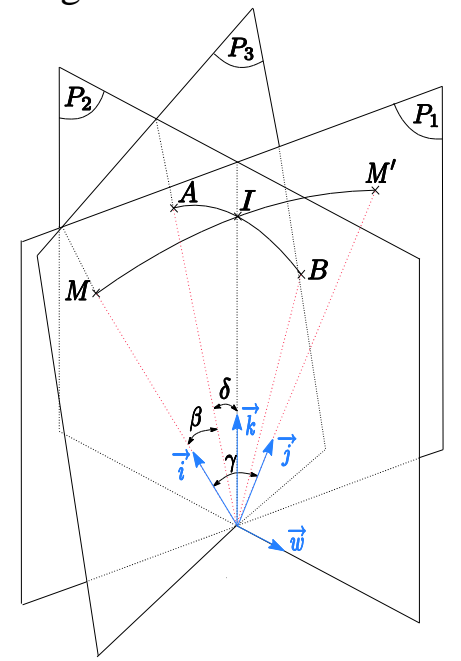

Figure 1: Main planes to define the meshing

Point $I$ denotes the intersection between the great circles $M M^{\prime}$ and $A B .\left(P_{3}\right)$ is the plane containing i and $\mathbf{a}$, we have $(\mathbf{i}, \mathbf{a})=\beta$. This angle is the image of the orthodromic distance between $M$ and $A$. 
Knowing the maximal speed of the vessel $V_{\text {Max }}$, imposed by the design of the hull and the power train characteristics, and the desired time of sailing $T_{\text {obj }}$, the maximal distance that can cross the vessel during the time window is :

$D_{\text {Max }}=T_{o b j} V_{\text {Max }}$

By this mean we can set the maximal distance on the great circle route $M A$ :

$M A=\frac{r D_{\text {Max }}}{2}$,

where $r$ is an dimensionless factor lower than the unit used to get some margin. From this equation (1), angle $\beta$ is calculable as follow:

$\beta=\frac{r T_{0 b j} V_{M a x}}{2 R}$,

with $R$ the Earth mean radius. To compute the value of $\delta$ angle, the following vectorial equations are used:

$a=\cos \delta k_{u}-\sin \delta w_{u}$

$b=\cos \delta k_{\mathrm{u}}+\sin \delta w_{w^{p}}$

$i . a=i .\left(\cos \delta k_{u}-\sin \delta w_{u}\right)$.

Developing this relation (5), one can write:

$\delta=\cos ^{-1}\left(\frac{\cos \beta}{\cos \left(\frac{Y}{2}\right)}\right)$,

$\left\{\begin{array}{l}\lambda=\tan ^{-1}\left(-\frac{y}{x}\right) \\ \phi=\sin ^{-1}\left(\frac{z}{R}\right)\end{array}\right.$.

Using the inverse spherical transformation (7) and the definition of $\mathbf{a}$ and $\mathbf{b}$ vectors $(3,4)$, it is possible to compute the spherical coordinates of $A$ and $B$ :

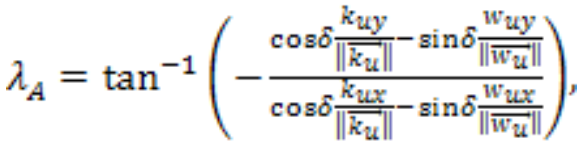

$$
\begin{aligned}
& \Phi_{A}=\sin ^{-1}\left(\cos \delta \frac{k_{u z}}{\left\|\overrightarrow{k_{u}}\right\|}-\sin \delta \frac{w_{u z}}{\left\|\overrightarrow{w_{u}}\right\|}\right) .
\end{aligned}
$$

Where $\lambda_{I}$ is the longitude of point $I$ and $\phi_{I}$ denotes its latitude. The same kind of relations can also be written for point $B$.

\subsection{Meshing's levels calculation}

The previous construction is extended in order to define the $N_{l}$ levels of the meshing. For that purpose, $i$ planes $P_{i}$ are defined, $i \in\left[1, N_{l}-2\right]$. These planes contain the vectors $\mathbf{w}$ and $\mathbf{k}_{i}$ (Fig. 2). The components of vector $\mathbf{k}_{i}$ are calculated according to:

$$
\left\{\begin{array}{l}
\text { i. } \mathbf{k}_{i}=\cos \left(i \frac{\gamma}{N_{\text {Lon }}-1}\right) \\
\text { i. } \mathbf{k}_{i}=0 \\
\mathbf{k}_{i} \cdot \mathbf{j}=\cos \left(\gamma-i \frac{y}{N_{\text {Lon }}-1}\right)
\end{array} .\right.
$$

By solving the system (10) the components of $\mathbf{k}_{i}$ are computed. To complete the spherical pyramid (Fig. 2), three more plans comparable to $\left(P_{3}\right)$ are defined : $\left(P_{4}\right)$ contains $\mathbf{i}$ and $\mathbf{b},\left(P_{5}\right)$ contains $\mathbf{j}$ and $\mathbf{a},\left(P_{6}\right)$ contains $\mathbf{j}$ and $\mathbf{b}$.

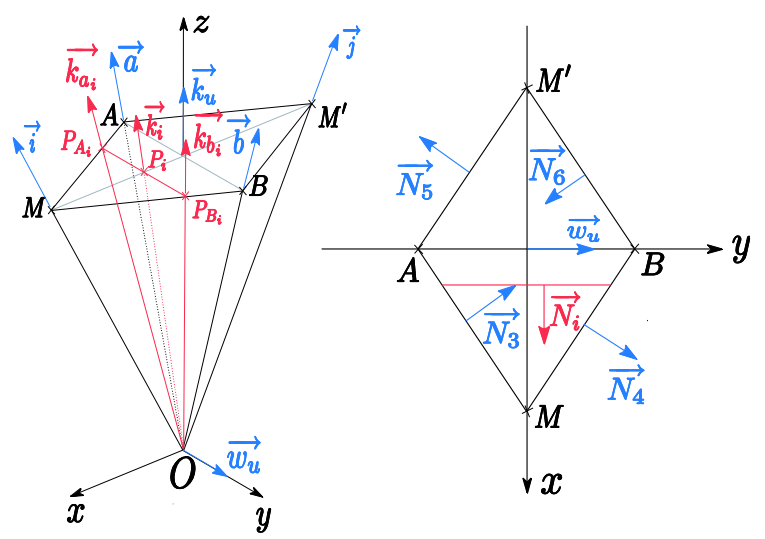

Figure 2: Definition of planes $P_{\mathrm{i}}$

For each of the above mention planes, their normal $\mathbf{N}_{3}, \mathbf{N}_{4}, \mathbf{N}_{5}, \mathbf{N}_{6}$ are defined, $\mathbf{N}_{i}$ the normal to $\left(P_{i}\right)$ is also calculated. The intersections between $\left(P_{i}\right),\left(P_{n}\right)$ and $\left(P_{n+1}\right)(n=\{3,5\})$ are the two lines called $\left(O P_{A_{i}}\right)$ and $\left(O P_{B_{i}}\right)$ oriented respectively by vectors $\mathbf{k}_{a_{\tilde{f}}}$ and $\mathbf{k}_{b_{\tilde{f}}}$. The components of these vectors are calculated according to :

- If $\left(\mathbf{i}, \mathbf{k}_{i}\right) \leq \frac{y}{2}$,

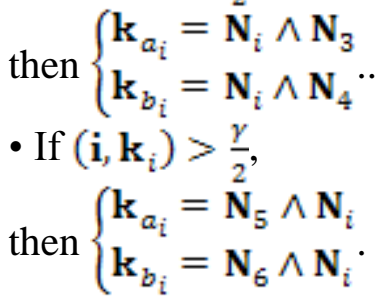

The related unit vectors $\mathbf{k}_{a_{i} u}$ and $\mathbf{k}_{b_{i} u}$ are defined. In each level of the meshing i.e. plane $P_{i}$ (Fig. 3), nodes of the level are defined. The distance between 2 nodes is constant and defined by the parameter $D_{L}$. As a result, in a level, $N_{\delta_{\tilde{j}}}$ nodes $P_{i, j}$ are defined with:

$N_{\delta_{i}}=\operatorname{int}\left(\frac{R \delta_{i}}{D_{L}}\right)+1$.

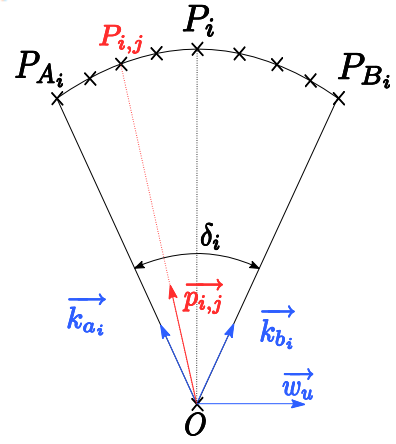

Figure 3: Vectors and nodes in plane $P_{\mathrm{i}}$ 
The angle $\delta_{i}$ between vectors $\mathbf{k}_{a_{\tilde{i}}}$ and $\mathbf{k}_{b_{\tilde{i}}}$ is defined by :

$\delta_{i}=\cos ^{-1}\left(\mathbf{k}_{a_{i} u^{x}} \mathbf{k}_{b_{i} w}\right)$.

For each node in the level, directing vector $\mathbf{p}_{i, j}$ of the line $\left(O P_{i, j}\right)$ is defined by the following system :

$$
\left\{\begin{array}{l}
\left(\mathbf{p}_{A_{i} w}, \mathbf{p}_{i, j}\right)=\cos \left(j \frac{\delta_{i}}{N_{\sigma_{i}}}\right) \\
\left(\mathbf{p}_{i, j}, \mathbf{p}_{B_{i} u}\right)=\cos \left(\delta_{i}-j \frac{\delta_{i}}{N_{\sigma_{i}}}\right) \\
\left(\mathbf{p}_{i_{i}, j}, \mathbf{N}_{i}\right)=0
\end{array}\right.
$$

So the cartesian coordinates of the $j^{\text {th }}$ node of the $i^{\text {th }}$ level are :

$\left\{\begin{array}{l}\mathbf{O P}_{i, j}=R \mathbf{p}_{j} \\ i \in\left[1, N_{l}-2\right] . \\ j \in\left[1, N_{\delta_{i}}\right]\end{array}\right.$

Using the inverse spherical transformation (7), the coordinates of $P_{i, j}(14)$ are expressed within the spherical coordinate system.

\subsection{Limitation of the possible nodes}

From vectorial cartographies, the matrix giving the depth of water according to longitude and latitude of meshing's points is compared with the draught of the boat. The nodes at which the depth is insufficient are removed from the grid (14).

A criterion of maximum course between two successive nodes is also defined. This criterion makes it possible to exclude the nodes which move the ship too away from its final destination $M^{\prime}$.

\section{ROUTE DEFINITION}

\subsection{Geographical definition of routes}

\subsubsection{Definition of routes}

Each possible route is defined using a navigable node of each level of the meshing. The nodes $\left(M, P_{1}, \ldots P_{N_{l}-2}, M^{\prime}\right)$ define the control points of the associated Bézier curve. We choose Bézier curve since it begins at $M$ and end at $M^{\prime}$ in addition the curve is always in the convex hull of the control polygon. For recall the Bézier curve is defined by :

$\sum_{i=0}^{\left(N_{l}-1\right)} \mathcal{B}_{i}^{\left(N_{l}-1\right)}(t) P_{i}, \quad t \in[0,1]$.

Where $\mathcal{B}_{i}^{\left(N_{L}-1\right)}(t)$ stand for the Bernstein basis polynomials.

\subsubsection{Discretization of Bézier curve}

Moreover because of its parametric definition the Bézier curve is discreditable. This discretization is done relatively to a parameter $N_{C C}$ corresponding to the maximal number of course changes per hour. The number of segments of a route $N_{C}$ is defined from the minimal distance i.e. the orthodromic distance $M M^{\prime}$ sailed at the maximum speed of the vessel $V_{\text {Max }}$ :

$N_{C}=N_{C C} \frac{d_{M M v_{\text {ortho }}}}{V_{\text {Max }}}$.

As a result, the points $P_{k}$ defining the route are computed as presented below :

$\left\{P_{k} \in \mathbb{R}^{2} \mid \sum_{i=0}^{\left(N_{l}-1\right)} B_{i}^{\left(N_{1}-1\right)}(t) P_{i=1} \quad t=\frac{k}{N_{c}} v \quad k=\left\{0_{x x x} N_{C}\right\}\right\}$.

The discretization of Bézier curves is done because on each facet of the route, we consider that both the weather and sea state remain constant. It also allows to define loxodromic courses between $P_{k}$ and $P_{k+1}$ which leads to a route defined by waypoints and courses. $N_{C C}$ must be set with great care because it corresponds to a compromise between the number of course changes that the captain has to perform and the approximation of the weather field along the course.

\subsection{Velocity along a route}

In order to locate the vessel both in time and space, the velocity on the course is set. As a result along each facet of the route, the time dependant weather data are known. Moreover the crossing time $T_{p_{k}, P_{k+1}}$ is easily calculable.

The target speeds of the vessel $V_{T}$ are included between two boundaries: $V_{T_{\text {Max }}}$ and $V_{T_{\text {Min }}} . V_{T_{\text {Min }}}$ has to be tuned by the captain. The number of target speeds is $N_{T S}$. Each target speed is valid on several segments of the discretized route. The number of facets $N_{S}$ on which $V_{T}$ is valid is defined by :

$N_{S}=\operatorname{int}\left(\frac{N_{C}}{N_{T S}}\right)+1$.

\subsection{Meteorological conditions along the route}

The weather and the sea state at the current position of the vessel are extracted from GRIB files defined with a regular $1.25^{\circ} \times 1^{\circ}$ grid downloaded from NOAA ftp ${ }^{1}$. These files gathered the meteorological data for a 180 hours time window with a 3 hours step.

${ }^{1}$ ftp://polar . ncep.noaa.gov/pub/waves/latest_run/ 
The decoding of these files allows the construction of true wind, current and waves fields for the sailing time window. The parameters of these fields are presented in Table 1.

Table 1: Parameters of weather and sea state

$\begin{array}{lll}V_{r} & \text { True wind speed } & m \cdot s^{-1} \\ \phi_{r} & \text { True wind direction } & \circ \\ V_{c} & \text { Current speed } & m \cdot s^{-1} \\ \Phi_{c} & \text { Current direction } & \circ \\ H_{w} & \text { Swell height } & m \\ T_{W} & \text { Swell period } & s \\ \Phi_{W} & \text { Swell direction } & \circ\end{array}$

As the meteorological grid weather does not correspond to the route's points $P_{k}$, a space interpolation of the data is done. For that purpose, we used 2D linear interpolation techniques to estimate the encountered conditions during the whole time window for each $P_{k}$. In addition, the time dependency of the fields can be easily taken into account by interpolation in time.

\section{MESHING SENSITIVITY TO ITS PARAMETERS}

The main advantage of the method compared to the previous approaches is that the rhombus definition is based on physical parameters (Tab. 2) easily adjusted and tuned by the captain. The journey is defined by $\mathbf{P}_{\text {Dep }}$ and $\mathbf{P}_{\text {Arr }}$. The design of the boat imposes $V_{\max }$ and the four other meshing's parameters define its geometry. On Fig. 4, two different values of each adjustable parameters are shown. The dots corresponds to the navigable nodes and the cross to the non navigable ones These parameters are left in user's hand but a great attention must be brought to their value since they define the manner the research space is discretized and then the fineness of the solution.

Table 2: Physical parameters defining the meshing

\begin{tabular}{lll}
\hline $\mathbf{P}_{\text {Dep }}$ & Departure point & Arrival point \\
$\mathbf{P}_{\text {Ary }}$ & Maximum speed of the vessel & $\mathrm{km} \cdot \mathrm{h}^{-1}$ \\
$V_{\max }$ & Objective time & $\mathrm{h}$ \\
$T_{\text {Obj }}$ & Number of levels & - \\
$N_{\mathbb{D}}$ & Distance between nodes & $\mathrm{km}$ \\
$D_{L}$ & Course changes per hour & $\mathrm{h}^{-1}$ \\
$N_{\text {CC }}$ &
\end{tabular}

Moreover the discretization of the research space is uniform because of the spherical definition of the meshing. This property is interesting for long voyages since the routes will be equi-distributed on
Earth's surface. Of course for short travels, the Earth's roundness is too small to have an influence on the meshing definition so a planar definition of the rhombus is more convenient.

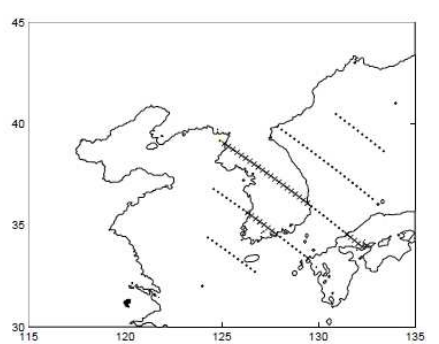

(a) $T_{O b j}=80 \mathrm{~h}$

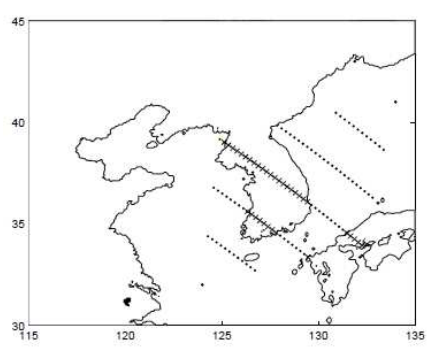

(c) $N_{l}=5$

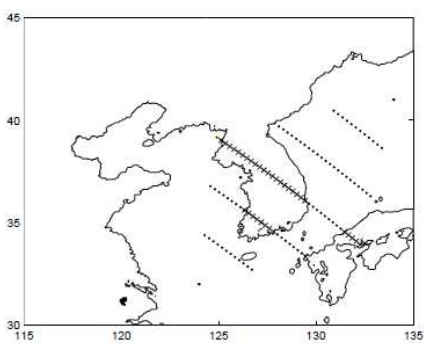

(e) $D_{L}=25 \mathrm{~km}$

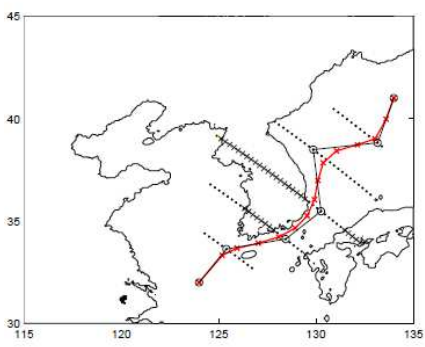

(g) $N_{C C}=1 / 4 h^{-1}$

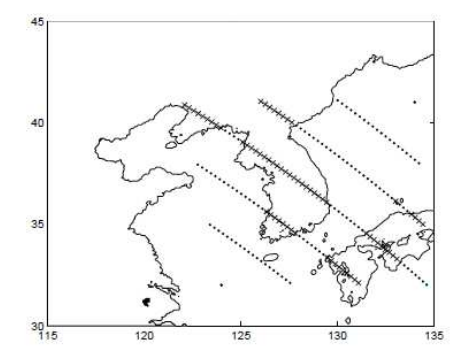

(b) $T_{O b j}=110 \mathrm{~h}$

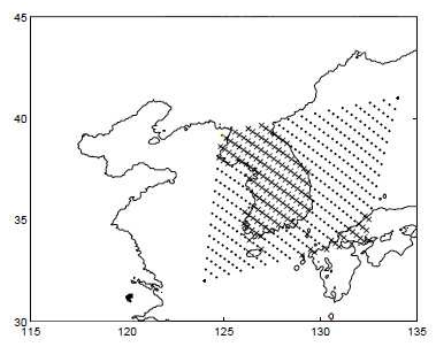

(d) $N_{l}=25$

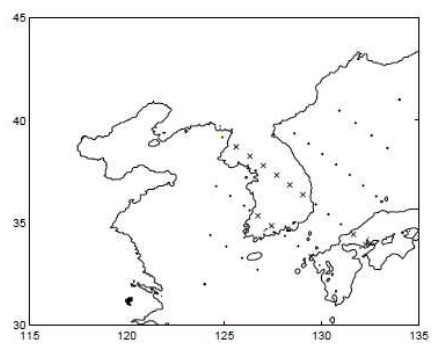

(f) $D_{L}=75 \mathrm{~km}$

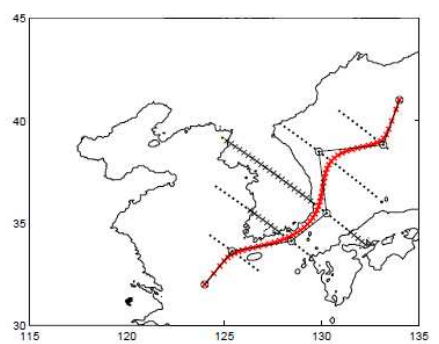

(h) $N_{C C}=1 h^{-1}$
Figure 4: Sensitivity to meshing's parameters

\section{CONSUMPTION MODELING}

At this point, the set of possible routes has been defined and the physical magnitudes have been calculated. In the perspective of optimizing the route of the vessel, a cost function has to be defined in order to select the best track.

In the literature, various approaches are proposed. For example Zappoli (1972) chose the sailing time 
as cost function, Hinnenthal \& al. (2003) chose both the consumption and the estimate time of arrival. But to compute these functions, the ship performances in a seaway must be accurately known Journée \& Mejiers (1980). Most of the works use parametric models in order to estimate the boat's behavior. The scheme of calculation in the recent approaches is often the same one :

- Estimation of the resistances acting on the hull :

- Still water resistance based on regression analysis of model tests and full-scale data (Haltrop, Van Oomertsen, ...),

- Wind resistance of the emmerged part of the vessel (Isherwood),

- Added resitance due to waves (Gerristma, Boese, ...),

- Operating point of the propeller with the ITTC power prediction method,

- Operating point of the main engine to estimate the consumption.

These parametric models are well known and controlled but their identification requires specific equipments such has wind tunnels or towing tanks, realization of instrumental models, instrumentation of the ship for full-scale measurements. The calculation time may be rather important and the measurement cost very high to get an efficient estimator of performances so applications of these methods are limited. Moreover the parameters are time dependent if we take into account the fouling of the hull leading to an increase of resistances for example. In addition, these models are not generic, the calculation method must be adapted to each hull's shape.

To analyze the efficiency of the proposed meshing method, we introduce a parametric model that will react to the encounter sea conditions. We are aware that such a raw model could not describe finely the resistances that encounter a boat in a real seaway but it as to be implanted to test the general approach we propose. The consumption model will further be identified on board using fuzzy logic technics. As the meshing method is uncoupled from the modeling, any function cost can be used to estimate the efficiency of a particular route. We used the ship presented in Hagiwara (1987) since many information are available and the time calculation cost is low ( $3 m s)$. For our routing scheme, we chose the consumption and the sailing time as estimators of the vessel efficiency.

\subsection{Scheme of calculation}

The scheme of consumption calculation is presented on the figure 5. As presented earlier, both the geography and the velocity are defined for a route, as a result, the inputs of the consumption calculation are the GPS position of the boat and the desired seabed velocities $V_{T}$.

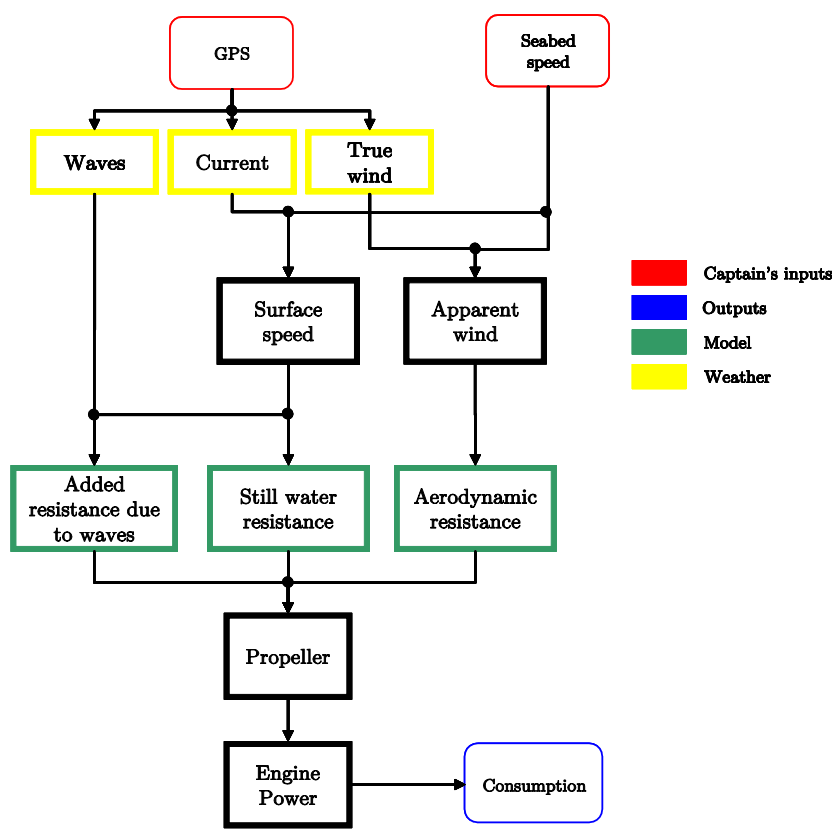

Figure 5: Scheme calculation of the consumption

Classically, the composition between true wind and seabed velocity of the vessel leads to the apparent wind. In order to get the desired seabed velocity, the current effects must be compensated if the resulting relative speed does not exceed the limitation due to the vessel design i.e. the maximal speed of the vessel $V_{\max }$. The drifting of the vessel is neglected here.

\subsection{Resistances computation}

In this part the relations used to compute the resistances acting on the hull are presented rapidly. They will allow to estimate the power that has to be delivered by the vessel's engine.

- Still water resistance $R_{S W}$ is the first resistance acting on the vessel. It corresponds to the energy used to overcome the frictional resistance of the hull plus the one used to create the bow wave.

- Aerodynamic resistance $R_{A}$ is the action of the wind onto the emerged part of the vessel.

- Added resistance due to waves $R_{A W}$ is the increase of resistance due to the encountered waves. It depends of their average period, significative height and mean direction. 
The total resistance $R_{T}$ is the global resistance acting on the hull. Its value is calculated according to:

$R_{T}=R_{S W}+R_{A}+R_{A W^{*}}$

The numerical values of the model parameters and the vessel design, are presented in Hagiwara \& Spaans (1987).

\subsection{Propulsion characteristics}

As the resistances acting on the hull are known, the propulsion system has to be modeled in order to evaluate the torque and power that must be delivered by the engine at the target velocity $V_{T}$. For the routing exemple we chose a $8 \mathrm{~m}$ diameter fixed pitch Wageningen B5-75 screw series propeller, Carlton (2007) and a $10000 \mathrm{~kW}$ Wärtsilä engine (2007).

Propeller thrust, torque, rate of revolution The propeller thrust $T_{p}$, torque $Q_{p}$ and power $P_{p}$ are calculated using the ITTC scheme of calculation (1978). Their calculation are well known and will not be discussed here. The propeller's rate of revolution is adjusted to obtain the proper thrust $T_{p}$.

Engine power and consumption For a known propeller and engine, a fixed propeller revolution rate and a given ship speed, the engine power can be calculated as follows:

$$
P_{e}=\frac{R_{T} V_{f}}{\eta_{H} \eta_{0} \eta_{X} \eta_{m s}}
$$

with $\eta_{H}=\frac{1-t}{1-w}$ the efficiency of the hull and $t$ the thrust deduction fraction due to suction of the water in front of the propeller, $w$ the wake fraction, $\eta_{0}$ the open water efficiency, $\eta_{r}$ the relative rotative efficiency and $\eta_{m}$ the mechanical efficiency of shaft bearing.

The consumption of the engine is calculated knowing the delivered power. For that purpose, we use the specific consumption law $C_{s}$ given by the engine manufacturer. For a given rate of revolution $N_{e}$ and power $P_{e}$ of the engine, the hourly consumption is given by :

$$
C=P_{e} C_{s}\left(N_{e}\right) \text {. }
$$

The unit of $P_{e}$ is $L \cdot h^{-1}$.

\section{OPTIMIZATION SCHEME}

\subsection{Search method}

\subsubsection{Performances indices}

The goal of the optimization is to minimize the antagonist objectives : the consumption $C$ and the sailing time $T$. A numerical optimization of a route off Corea is presented hereafter (Fig. 6). The journey is defined between $\mathbf{P}_{D \beta p}=\left(133^{\circ} 40^{\circ}\right)$ and $\mathbf{P}_{\text {Arr }}=\left(122.5^{\circ} 32.5^{\circ}\right)$. The number of level is $N_{l}=7$, the distance between nodes of a level is $D_{L}=25 \mathrm{~km}$ and the number of course changes per hour is $N_{C C}=1 h^{-1}$. The number of target speeds is $N_{T S}=8$. For this application we used the meteorological data of the $23^{\text {th }}$ April 2008 at 0:00 GMT which will be the departure time.

\subsubsection{Pareto-optimal solutions}

Solving this optimization problem with conflicting objectives across a high-dimensional research space is a difficult goal. Instead of a single optimum, there is rather a set of alternative tradeoffs, generally known as Pareto-optimal solutions. Various evolutionary approaches to multi-objective optimization have been proposed since 1985, capable of searching for multiple Pareto-optimal solutions concurrently in a single simulation run , Valdhuizen \& Lamont (2000). The optimization program FRONTIER ${ }^{\circledR 2}$ and the technical computing software MATLAB ${ }^{\circledR}$ are used to set up the framework of the multi-objective design optimization study of weather routing. The Multiobjective Genetic Algorithm (MOGA), implemented first by Fonseca \& Fleming (1998), is used to perform the optimization problem.

\subsubsection{Design parameters}

The number of parameters necessary to define a route is $\left(N_{l}-2+N_{T S}\right)$. For each level of the meshing the associate parameter is the index $j$ of the node $P_{i, j}$. Concerning the target speeds, the parameters are within the boundary previously presented and their step is $0.1 \mathrm{kn}$.

\subsubsection{Global optimization process}

The algorithm will attempt a number of evaluations equal to the size of the initial population for the MOGA multiplied by the number of

\footnotetext{
${ }^{2}$ http://www.esteco.com/
} 
generation. The initial population is generated by a random sequence of 60 designs. The major disadvantage of the MOGA is mainly related to the number of evaluations necessary to obtain satisfactory solutions. The search for the optimal solutions extends in all the directions from design space and produces a rich data base and there is not a true stop criterion. The numerical evaluation of the performances calls upon MATLAB codes is not so expensive in terms of computing time (about $2 s$ ). In an attempt to solve the optimization problem in an acceptable timeframe, the number of generations evaluated is almost 70, i.e. 4000 designs in all. The required computation time for the global optimization process is about 2 hours $(2.4 \mathrm{GHz} / 3.0$ $\mathrm{Gb}$ RAM). Integrating a Response Surface Methodology to reduce the computation time could be an interesting extension of our work especially if one wants to achieve on board routing.

\subsection{Numerical optimizations}

The time dependency of the sea state and wind field is taken into account with linear interpolation in time. Figure 6 presents an exemple of weather routing. On these maps, two positions of the vessel are shown. For the analysis of the optimization, we compute the shortest path and we tune the constant velocity on this path so that the sailing time is the same as the optimal one. The comparison between these two routes is done in Table 3
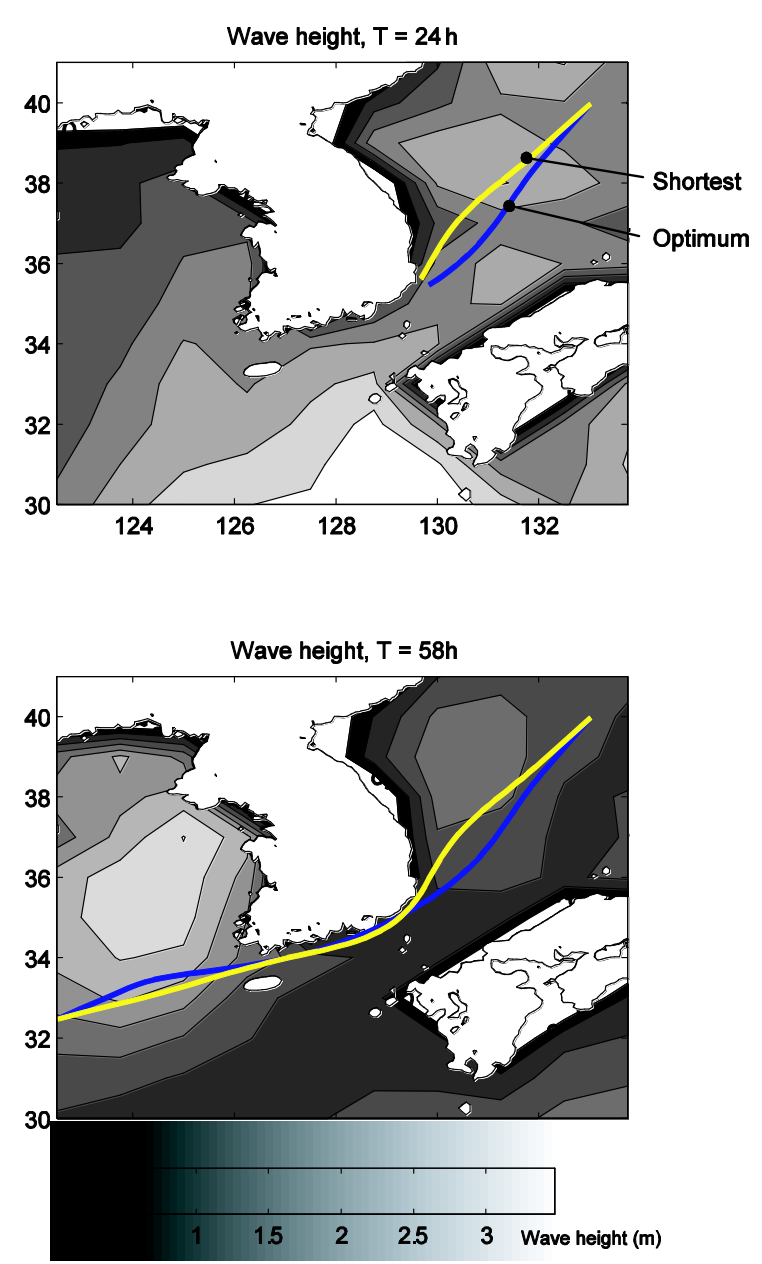

Figure 6: Comparison between shortest and optimal routes in wave field

Table 3: Comparison of the optimum route to the shortest one

\begin{tabular}{llll}
\hline Route & Distance $(\mathrm{km})$ & Time $(h)$ & Fuel $(t)$ \\
\hline Shortest & 1337.7 & 56.8 & 198.4 \\
Optimal & 1342.1 & 56.8 & 188.4
\end{tabular}

This example puts forward the interest of the routing weather for motor vessel. We have shown that a longer distance of journey does not imply automatically an increase of consumption. The example above shows that a saving of $5 \%$ is realizable on the chosen journey. The economy are not very large because the distance to be traversed is too short. Moreover, the sea is not rough, the maximum height of waves during the journey is only $2 m$. Another problem that might occur is the spatial interpolation of the conditions. In the NOAA's GRIB, on earth, the fields are not known so an arbitrary value is set (for instance 0). This unknowing of the fields leads to inconsistent value of the advancing resistance along the coast that might distorted the results. 


\section{CONCLUSION \& FUTURE WORK}

This paper presents a brief outlook to motor vessel routing using deterministic weather forecasts. A method for spatial and temporal generation of route variants based on a generic and automatic meshing method has been presented. The major advantage of this technic is the physic based definition of the rhombus and the low number of free variables used to define a route. The ship route was optimized using multi-objective algorithm for a deterministic weather case. The reasonable computation time will allow the use of this method for on board routing applications. Paretooptimization may be considered as a tool providing a set of efficient solutions among different and conflicting objectives, under different constraints. The final choice remains always subjective and is let to the user's hands.

Future works concern the improvement of the consumption prediction model using on board measurements and fuzzy logic identification technics. We will also investigate the routing of wail-assisted motor vessels. The use of these technics will allow the quick establishment of reliable models of different types of vessels keeping the measurement cost very low since no tawing tank, wind-tunnel tests or models will be necessary. An other aspect that has not been discussed in this paper is the stochastic nature of weather and sea conditions that must been taken into account. This aspect will also be investigated using robust optimization technics. The reactualization of the routing policy during the journey will also be considered for long travels. Thus we will be able to integrate the most recent weather data.

\section{ACKNOWLEDGEMENTS}

The research in this paper is supported by the Région Bretagne and some local community. This work takes place in the Grand Largue project.

\section{REFERENCES}

Allsopp, T. 2000. Optimising Yacht Routes Under Uncertainty. Proceedings of the 2000 Fall National Conference of the Operations Reaseach Society of Japan : 176-183.

Bleick, W. \& Faulkner F. 1965. Minimum-Time Ship Routing. Journal of Applied Meteorology, 4 : 217-221.

Braddock, R.D. 1970. On Meteorological Navigation. Journal of Applied Meteorology 9(1) : 149-153.
Böttner, C.U. 2007. Weather Routing for Ships in Degraded Condition. International Symposium on Maritime Safety, Security and Environmental Protection, Athens, Greece.

Carlton, J. 2007. Marine Propellers and Propulsion. Butterworth-Heinemann.

Fonseca, C.M. \& Fleming, P.J. 1998. Multiobjective Optimization and Multiple Constraint Handling with Evolutionary Algorithms. IEEE Trans. On Systems, Man and Cybernetics $28: 26-37$.

Hagiwara, H. \& Spaans, J. 1987. Practical Weather Routing of Sail-Assisted Motor Vessels. Journal of Navigation 40(1) : 96-119.

Haltiner, G.J., Hamilton, H.D. \& Árnason, G. 1962. MinimalTime Ship Routing. Journal of Applied Meterology 1(1) : 17.

Harries, S., Heinmann, J. \& Hinnenthal J. 2003. ParetoOptimal Routing of Ships. International Conference on Ship and Shipping Research.

Hinnentham, J. \& Saerta, Ø. 2005. Robust Pareto-Optimal Routing of Ships Utilizing Ensemble Weather Forecasts. Maritime Transportation and Exploitation of Ocean and Coastal Resources : 1045-1050.

ITTC. 1978. 1978 ITTC Performance Prediction Method for Single Screw Ships. Proceedings of the $15^{\text {th }}$ International Towing Tank Conference, 1978, The Hague, Netherland.

James, R.W. 1957. Application of Wave Forecasts to Marine Navigation. U.S. Navy Hydrographic Office.

Journée, J.M.J. \& Mejijers, J.H.C. 1980. Ship Routeing for Optimum Performance. IME Transactions : 1-17.

Wärtsilä. 2007. Project Guide Wärtsilä 46.

Valdhizen, D.A.V. \& Lamont, G.B. 2000. Multiobjective Evolutionary Algorithms : Analyzing the State-of-the-art. Evolutionary Computation 8 : 125-147.

Zappoli, R. 1972. Minimum-Time Routing as an N-Stage Decision Process. Journal of Applied Meteorology 11(3) : 429-435. 